Cite this article: Miricescu D, Totan A, Calenic B, Mocanu B, Greabu M. Salivary and serum cortisol in patients with periodontal disease and oral lichen planus. Stoma Edu J. 2015;2(1):51-56

\section{SALIVARY AND SERUM CORTISOL IN PATIENTS WITH PERIODONTAL DISEASE AND ORAL LICHEN PLANUS}

https://doi.org/10.25241/stomaeduj.2015.2(1).art.5

\section{Daniela Miricescu ${ }^{1 \mathrm{a}}$ Alexandra Totan ${ }^{16}$, Bogdan Calenic ${ }^{1 c}$, Brânduşa Mocanu ${ }^{2 d}$ Maria Greabu ${ }^{1 e^{*}}$}

1. Department of Biochemistry Faculty of Dental Medicine, "Caro Davila" University of Medicine and Pharmacy, Bucharest, Romania 2. Department of

Periodontology, Faculty of Denta Medicine, "Carol Davila" University of Medicine and Pharmacy, Bucharest, Romania

a. PhD, Teaching Assistant b. PhD, Lecturer c. PhD, Lecturer

d. PhD, Teaching Assistant e. PhD, Professor, Head of Department
Received: May, 4 th 2015 Accepted: June, 1st 2015

* Corresponding author: Professor Maria Greabu, DDS, PhD Address: 8 Blvd Eroii Sanitari, Bucharest Romania; Telephone: +40721274932 R0-050474, Fax:021 3110984, E-mail: maria greabu@yahoo.com

\section{Abstract}

Introduction. Periodontitis and oral lichen planus are two oral diseases that affect the adult population worldwide.

Aim: A positive relationship had been proposed between psychological stress, periodontitis and oral lichen planus. The general aim of our study was to investigate the levels of "cortisol", the stress- related hormone in patients with periodontal disease and oral lichen planus.

Methodology. Our study included 20 patients with chronic periodontitis, 20 with oral lichen planus and 20 healthy subjects. We collected non-stimulated whole saliva and serum from patients and healthy subjects. Serum and salivary cortisol was measured using the ELISA method.

Results. In both patients with chronic periodontitis and oral lichen planus, we obtained, in statistical terms, increased levels of salivary and serum cortisol versus the control group $\mathrm{p}<0.05$.

Discussions. Psychological stress may influence the evolution and progression of this two oral diseases. Cortisol can be regarded as the most useful biomarker to evaluate stress.

Conclusions. Psychological stress may be regarded as risk factor for periodontal disease and oral lichen planus progression.

Keywords: psychological stress, oral diseases, cortisol, biomarker

\section{Introduction}

Periodontitis, is a nonreversible inflammatory disease affecting the tooth supporting tissues. This chronic disease is caused by pathogenic microorganisms, three organisms in particular, Tanerella forsythensis, Porphyromonas gingivalis, and Treponema denticola, which have been directly associated with chronic periodontitis (1-3). Others factors can influence the appearance and evolution of this disease are oxidative stress and psychological stress (4-8). Psychological stress may be involved in the inflammatory responses and changes in the composition of dental biofilm (9). Oral lichen planus (OLP), a chronic inflammatory mucutaneous disease of unknown etiology, affects mainly the adult population (approximately 0.02-
4\%). In the pathogenesis of OLP genetic infectious, autoimmune factors (10-12) seem to be involved.

Cortisol, the "stress hormone" is the most widely-used biomarker in stress evaluation studies. Among other fluids the free fraction or the biological active form can be readily detected in saliva (13). Saliva is currently used as a diagnostic tool for systemic and oral diseases, because of its numerous advantages: easy to collect, non-invasive technique and no special equipment for collection $(14,15)$.

The aim of our study was to detect salivary and serum levels of cortisol in patients with chronic periodontitis and OLP. To the best of our knowledge this is the first study to assess salivary cortisol levels in both periodontitis and oral lichen planus patients. 


\section{Materials and methods Periodontal disease patients}

The study included twenty patients with chronic periodontitis ( 5 males and 15 females, with an average age of $51.26 \pm 7.4)$. The ethics board of "Carol Davila" University of Medicine and Pharmacy, Faculty of Dental Medicine, reviewed and approved our study. All clinical examinations were performed by one qualified examiner from the Department of Periodontology. Classical clinical parameters were measured: PD (probing depth), plaque index (PI), bleeding index (GI). GI and $\mathrm{PI}$ are expressed as percentage (\%).

\section{Oral Lichen Planus patients:}

20 patients with OLP (clinical type: Keratosis and Atrophic/erosive lesions) including 15 males; 5 females aged between 18-68 years. The clinical examinations were performed at the Oral Pathology Department, Faculty of Dental Medicine, "Carol Davila" University of Medicine and Pharmacy, Bucharest. Twenty healthy subjects with no gingival inflammation, good oral hygiene and no history of periodontal disease and OLP made up the control group.

\section{Saliva and blood collection:}

Non-stimulated whole saliva has been collected between 9 and 10 a.m, $(1.0-2.0 \mathrm{ml})$. Saliva collected in sterile test tube was centrifuged at 3000 rpm for $10 \mathrm{~min}$. At the same time $5 \mathrm{ml}$ of blood were collected and the serum was immediately obtained. Both saliva and serum cortisol were detected using the ELISA method.

Salivary and serum cortisol assays: A microtitre plate is coated with monoclonal antibodies to cortisol. Cortisol in standards and unknowns competes with cortisol linked to horseradish peroxidase (HRP) for the antibody binding sites. After incubation, unbound components are washed away. Bound cortisol peroxidase is measured by the reaction of the peroxidase enzyme with the substrate tetramethylbenzidine (TMB). This reaction generates a blue color. After stopping the reaction with sulfuric acid, a yellow color is generated. We read the optical density on a standard plate reader at $450 \mathrm{~nm}$. The amount of cortisol peroxidase detected is measured by the intensity of its color and is inversely proportional to the amount of cortisol present.

\section{Statistical analysis}

Data distributions were expressed as means, standard deviations (SD), ranges, and percentages, as appropriate. The Pearson's correlation coefficient and ANOVA test were used. A p-value $<0.05$ was considered statistically significant.

\section{Results}

Our results reflect statistically increased salivary levels in patients with periodontal disease and OLP compared with controls.

Increased serum levels for cortisol were obtained in patients with periodontal disease and OLP

Table 1. Mean values for salivary cortisol in patients with periodontal disease

\begin{tabular}{|c|c|c|c|}
\hline Parameter & Patients & Control group & \\
\hline Cortisol & $10,1 \pm 1,03$ & $3,83 \pm 0,87$ & $<0.0001$ \\
\hline $\begin{array}{c}\text { Periodontal disease } \\
\text { patients } \\
\text { (ng/mL) }\end{array}$ & $10,13 \pm 1,056$ & $3,83 \pm 0,87$ & $<0.0001$ \\
\hline $\begin{array}{c}\text { OLP patients } \\
\text { (ng/mL) }\end{array}$
\end{tabular}

Table 2. Mean values for serum cortisol in patients with periodontal and OLP versus controls

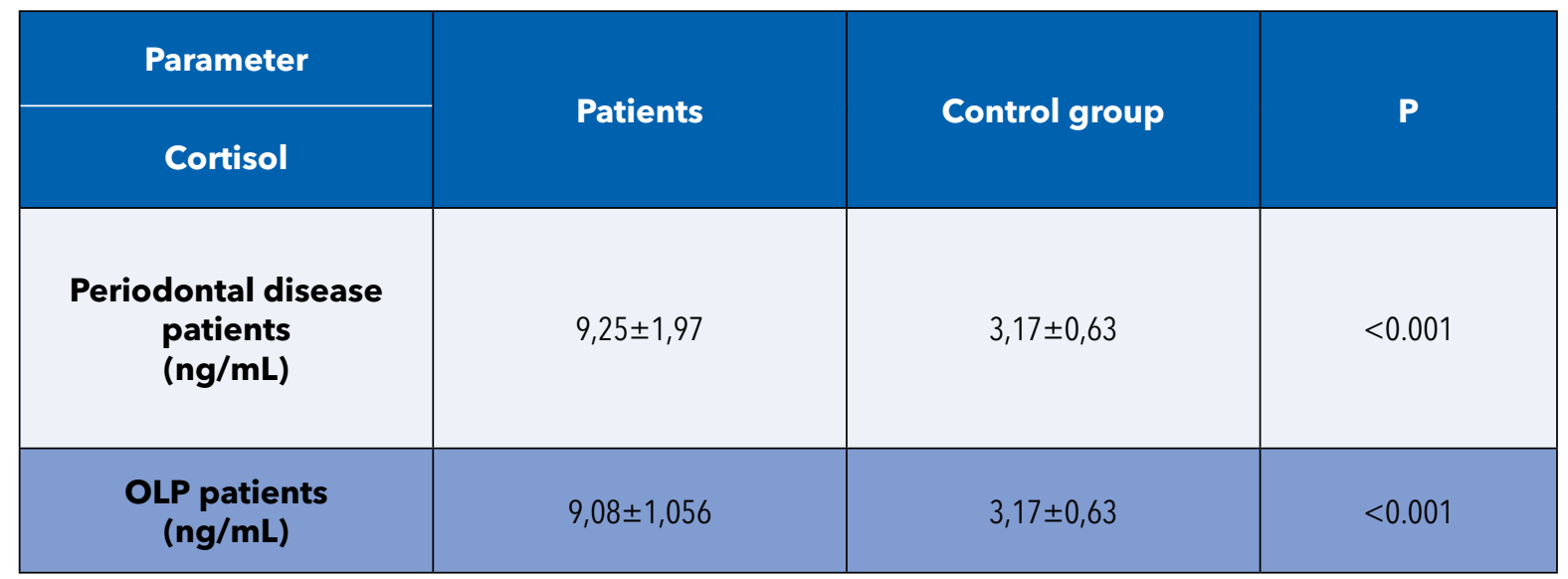


versus healthy subjects.

\section{Discussion}

Saliva is an excellent natural ultrafiltrate that contains local substances as well as others derived from the blood. In saliva we can detect a myriad markers such as: cytokines (TNF- $\alpha$, IL-6, IFN- $\gamma$, MIP-1 $\beta$ ), C Reactive Protein (CRP), antioxidant biomarkers (uric acid, malondialdehyde, antioxidant enzymes, total antioxidant capacity) insulin resistance markes (adipokines) or cortisol $(13,14,16)$.

Steroids, such as cortisol, not bound by carrier proteins, can diffuse freely into saliva, being the free fraction. The concentration of cortisol in saliva is independent of the salivary flow rate and strongly correlated with circulating cortisol concentration. In the reference literature, cortisol is the most common used indicator of stress, released by the hypothalamo-pituitary-adrenal (HPA) axis, under the influence of several factors such as chronic inflammation $(13,17)$.

The most important effects of cortisol release are: supression of the inflammatory response, modifying cytokine profiles, elevation of blood glucose levels and alteration of certain growth factors levels(18, 19). Low levels of cortisol were detected in patients with primary adrenal insufficiency (Addison's disease) and ACTH deficiency $(20,21)$. Higher levels of cortisol were found in patients with systemic (Cushing's disease, malignancy) and oral diseases (22-34).
In healthy subjects, cortisol is higher in the morning (0.20-1.41 $\mu \mathrm{g} / \mathrm{mL})$, compared to the afternoon values $(0.04-0.41 \mu \mathrm{g} / \mathrm{mL})$. Salivary concentrations reflect the activity of HPA axis (35) very well. Salivary cortisol levels reflect endocrine abnormalities, insulin resistance, hypertension, dyslipidemia and type 2 diabete (13).

Previous studies reported higher levels of this biomarker in patients' saliva. Salivary cortisol was detected from stimulated, unstimulated whole saliva and from gingival crevicular fluid. The results were the same: higher levels of cortisol in all different forms of periodontitis (aggressive, chronic periodontitis). Anxious patients with chronic periodontitis present higher levels of cortisol and anxiety. Anxiety may be regarded as an important factor in the progression of periodontal diseases (8, 9, 25-30).

In our study we detected cortisol from unstimulated whole saliva and serum and detected increased levels with patients with chronic periontitis versus healthy subjects.

During periods of stress it is believed that oral hygiene is neglected and is associated with attachment loss and missing teeth. Cortisol exercise inhibitory effects on the inflammatory immune response, because IL-12 is inhibited and IL-10 is stimulated by macrophages. These changes have major effects on the immune and inflammatory response and conduce to increased

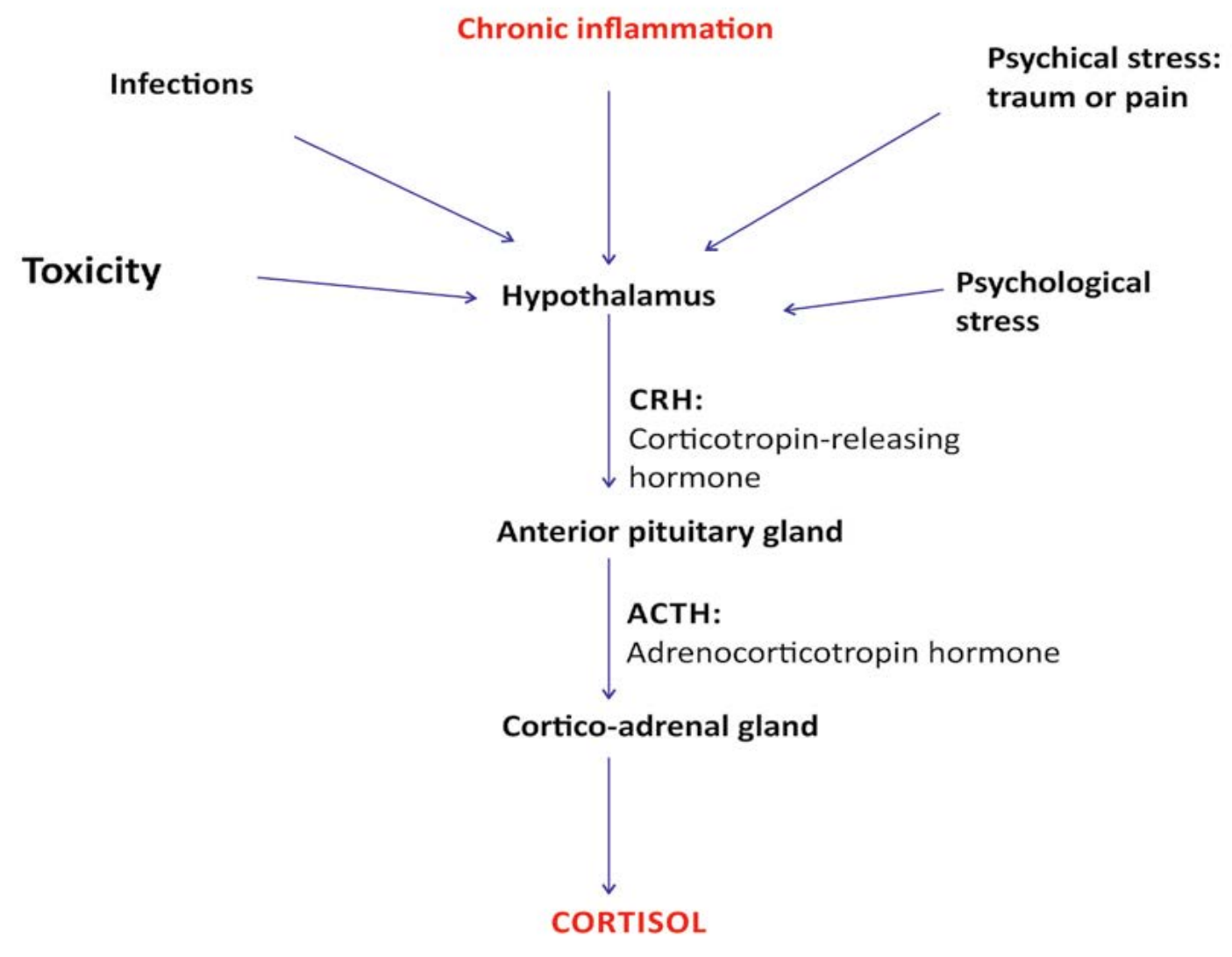

Figure 1. Cortisol release factors by HPA axis (17) 
susceptibility to pathogenic microorganisms $(18,19$, 36).

Positive correlation between stress and cortisol, stress and clinical parameters, and cortisol levels and patients with chronic periodontitis had been observed by Goyal $S$ et al (30). At the same time Shah et all, observed a positive correlation between psychological factors and salivary cortisol levels in the OLP patients (34). Giardini and co-workers, indicated an association between OLP and anxiety, but salivary cortisol levels did not differ between patients with OLP and control group (33).

In our study, salivary and serum levels of cortisol were statistically increased in OLP patients versus healthy subjects. Until now there are no studies regarding the serum level of cortisol at patients with OLP. The World Health Organisation (WHO) classifies OLP as a "potentially malignant disorder" and suggests that OLP patients should be under close monitoring. OLP patients present higher levels of anxiety, depression because there are concern about the malignant transformation of this oral diseases (37).

Chronic inflammation leads to cortisol release that counteracts inflammatory reactions and even immunological functions, so this stress hormone can be involved in the pathogenesis of OLP and periodontal disease. In conclusion psychological stress may influence the progression of these two oral diseases.

\section{Acknowledgements}

This study was supported by the Sectorial Operational Programme Human Programme Human Resources Development (SOP HRD), financed from the European Social Fund and by the Romanian Government under the contract number POSDRU/6/1.5/S/S17.

\section{Bibliography}

1. Page RC, Kornman KS. The pathogenesis of human periodontitis: an introduction. Periodontol 2000.1997;14(1):9-11.

2. Ridgeway EE. Periodontal disease: diagnosis and management. J Am Acad Nurse Pract. 2000;12(3):79-84.

3. Miricescu D, Totan A, Calenic B, Mocanu B, Didilescu A, Mohora M, Spinu T, Greabu M. Salivary biomarkers: Relationship between oxidative stress and alveolar bone loss in chronic periodontitis. Acta Odonto Scand. 2014;72(1):42-47.

4. Su H, Gornitsky M, Velly AM, Yu H, Benarroch M, Schipper HM. Salivary DNA, lipid, and protein oxidation in nonsmokers with periodontal disease. Free Radic Biol Med. 2009;46(7):914-921.

5. Takane M, Sugano N, Ezawa T, Uchiyama T, Ito K. A marker of oxidative stress in saliva: association with periodontally-involved teeth of a hopeless prognosis. J Otal Sci. 2005;47(1):53-57.

6. D'Aiuto F, Nibali L, Parkar K, Patel, Suvan J, Donos N. Oxidative stress, systemic inflammation and severe periodontitis. J Dent Res. 2010; 89(11):1241-1246.

7. Miricescu D, Greabu M, Totan A, Mohora M, Didilescu A, Mitrea N, Arsene A, Spinu T, Totan C, Rădulescu R. Oxidative Stress - A possible link between systemic and oral diseases. Farmacia. 2011;6(3):329-337.

8. Mannem S, Chava VK. The effect of stress on periodontitis: A clinic-biochemical study. J Indian Soc Periodontol. 2012;16(3):365-369.

9. Akcali A, Huck O, Tenenbaum H, Davideau JL, Buduneli N. Periodontal diseases and stress: a brief review. J Oral Rehabil. 2013;40(1):60-68.
10. Totan A, Miricescu D, Parlatescu I, Mohora M, Greabu M. New possible salivary and serum biomarkers in oral lichen planus. Biotech Histochem. 2015;3:1-7.

11. Sugerman PB, Savage NW, Walsh LJ, Zhao ZZ, Zhou XJ, Khan A, Seymour GJ, Bigby M. The pathogenesis of oral lichen planus. Crit Rev Oral Biol Med. 2002; 13(4):350-365.

12. Farhi D, Dupin N. Pathophysiology, etiologic factors, and clinical management of oral lichen planus, part I: facts and controversies. Clin Dermatol 2010;28(1):100-108.

13. Greabu M, Purice M, Totan A, Spînu T, Totan C. Salivary cortisol-marker of stress response to different dental treatment. Rom J Intern Med. 2006;44(1):49-59.

14. Greabu $M$, Battino $M$, Mohora $M$, Totan A, Didilescu A, Spinu T, Totan C, Miricescu D, Radulescu R. Saliva--a diagnostic window to the body, both in health and in disease. J Med Life. 2009; 2(2):124-132.

15. Kaufman E, Lamster I. The diagnostic applications of saliva-a review. Int American Assoc Dent Res. 2002;13(2):197-221.

16. Desai GS, Mathews ST. Saliva as a non-invasive diagnostic tool for inflammation and insulin resistance. World J Diabetes. 2014;15:5(6):730738.

17. Greabu M, Totan A. Tratat de Chimie şi Biochimie pentru Medicină Dentară. Bucureşti: Ed. Standardizarea; 2011.

18. Miller DB, O'Callaghan JP. Neuroendocrine aspects of the response to the stress. Metabolism. 2002;51(6 Suppl 1):5-10. 
19. Takada T, Yoshinari N, Suguushi S, Kawase H, Yamane T, Noguchi T. Effect of restrain stress on the progression of experimental periodontitis in rats. J Periodontol. 2004;75(2):306-315.

20. Ekman B, Blomgren J, Adersson PO, Carlsson M, Arnqvist HJ. Variable sensivity to the glucocorticoid activity of cortisol in patients with primary adrenal insuffiency: assessment with ACTH profiles. Horm Metab Res. 2010;42(13):961-966.

21. Luvask K, Thotsen TE, Husebye ES. Saliva cortisol measurement: simple and reliable assessment of the glucocorticoid replacement therapy in Addison's disease. J Endocrinol Invest. 2006;29(8):727-731.

22. Lindsay JR, Oldfield EH, Stratakis CA, Nieman LK. The postoperative basal cortisol and $\mathrm{CRH}$ tests for prediction of long-term remission from Cushing's disease after transphenoidal surgery. J Clin Endocrinol Metab. 2011;96(7): 2057-2064.

23. Monteith SJ, Strake RM, Jane JA Jr, Oldfield $\mathrm{EH}$. Use of histological pseudocapsule in surgery for Cushing disease: rapid postoperative cortisol decline predicting complete tumor resection. J Neurosurg. 2012;116(4):721-727.

24. Dedert E, Lush E, Chagpar A, Dhabhar FS, Segerstrom SC, Spiegel D, Dayyat E, Daup M, McMasters K, Septhton SE. Stress, coping and circadian disruption among women awaiting breast cancer surgery. Ann Behav Med. 2012; 44(1):10-20.

25. Nayak SU, Nayak DG, Uppoor AS, Pai KK. Evaluation of cortisol levels in gingival crevicular fluid and saliva in anxious and non-anxious patients with chronic periodontitis. Dent Res J (Isfahan). 2013;10(4):474-481.

26. Rai B, Kaur J. Periodontal status, salivary immunoglobulin, and microbial counts after short exposure to an isolated environment. J Oral Sci. 2013; 55(2):139-143.

27. Haririan H, Bertl K, Laky M, Rausch WD, Böttcher M, Matejka M, Andrukhov O, Rausch-Fan X. Salivary and serum chromogranin $A$ and $\alpha$-amylase in periodontal health and disease. J Periodontol.
2012;83(10):1314-1321.

28. Rai B, Kaur J, Anand SC, Jacobs R. Salivary stress markers, stress, and periodontitis: a pilot study. J Periodontol. 2011; 82(2):287-292.

29. Rosania AE, Low KG, McCormick CM, Rosania DA. Stress, depression, cortisol, and periodontal disease. J Periodontol. 2009;80(2):260-266.

30. Goyal S, Jajoo S, Nagappa G, Rao G. Estimation of relationship between psychosocial stress and periodontal status using serum cortisol level: a clinico-biochemical study. Indian J Dent Res. 2011;22(1):6-9.

31. Nadendla LK, Meduri V, Paramkusam G, Pachava KR. Association of salivary cortisol and anxiety levels in lichen planus patients. J Clin Diagn Res. 2014; 8(12):ZC01-3.

32. Pippi R, Patini R, Ghiciuc CM, Sandu RB, Pasquali $V$, Scaccianoce S, Dima-Cozma LC, Patacchioli FR. Diurnal trajectories of salivary cortisol, salivary $\alpha$-amylase and psychological profiles in oral lichen planus patients. J Biol Regul Homeost Agents. 2014;28(1):147-154.

33. Girardi C, Luz C, Cherubini K, de Figueiredo MA, Nunes ML, Salum FG. Salivary cortisol and dehydroepiandrosterone (DHEA) levels, psychological factors in patients with oral lichen planus. Arch Oral Biol. 2011;56(9):864-868.

34. Shah B, Ashok L, Sujatha GP. Evaluation of salivary cortisol and psychological factors in patients with oral lichen planus. Indian J Dent Res. 2009;20(3):288-292.

35. Bozovic D, Racic M, Ivkovic N. Salivary cortisol levels as biological marker of stress reaction. Med Arch. 2013; 67(5):374-377.

36. Mengel $R$, Bacher $M$, Flores-de-Jacoby $L$. Interactions between stress, interleukin-1 $\beta$, interleukin-6 and cortisol in periodontally diseased patients. J Clin Periodontol. 2002;29(11): 1012 1022.

37. Warnakulasuriya S, Johnson NW, van der Waal I. Nomenclature and classification of potentially malignant disorders of the oral mucosa. J Oral Pathol Med. 2007;36(10): 575-580. 


\section{Daniela Miricescu}

Teaching Assistant, PhD Department of Biochemistry, Faculty of Dental Medicine "Carol Davila" University of Medicine and Pharmacy Bucharest, Romania

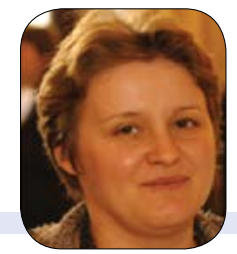

Her PhD, focused on the study oxidative stress as a new pathological biochemical mechanism in oral diseases, using saliva as a new

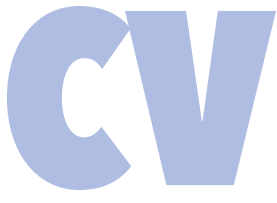
diagnostic fluid in oral diseases.

In the spring of 2011, during an exchange program, worked at Università Politecnica delle Marche, Italia, under the direct supervision of Maurizio Battino, PhD, DSc, MS, MD (Hon), focusing on the biochemical mechanisms of oxidative stress and antioxidants.

Her current research interests are, oxidative stress, oral stem cells properties, nanoparticles, and their possible interaction with the oral stem cells and generation of oxidative stress.

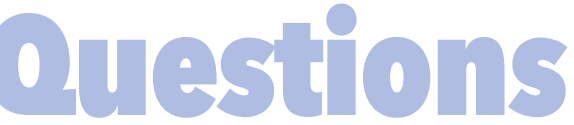

\section{Regarding this study:}

$\square$ a. The general aim of our study was to investigate the levels of cortisol in stimulated whole saliva b. 50 patients were included in our study

$\square$ c. Psychological stress may influence the evolution and progression of periodontal disease and oral lichen planus

d. Salivary cortisol was measured using RT-PCR method

\section{Regarding the patients included in our study:}

a. 1.0-2.0 of non-stimulated whole saliva and $5 \mathrm{ml}$ of blood were collected from patients

b. Optical density was read at $550 \mathrm{~nm}$

c. 40 patients with OLP with the age between 61-68 years

$\square$ d. 15 males and 5 females formed the patients with periodontal disease

\section{Regarding the salivary levels:}

$\square$ a. All the results were statistically decreased

b. The cortisol at patients with periodontal disease was $10.1 \pm 1.03$

c. The cortisol at patients with oral lichen planus was $9.08 \pm 1.056$

$\square \mathrm{d}$. The cortisol at patients with periodontal disease was $3.17 \pm 0.63$

\section{Regarding the serum levels:}

a. Levels for cortisol were not statistically significant

$\square$ b. The cortisol at patients with periodontal disease was $3.17 \pm 0.63$

$\square$ c. The cortisol at patients with oral lichen planus was $9.25 \pm 1.97$

$\square \mathrm{d}$. The cortisol at patients with oral lichen planus was $9.08 \pm 1.056$ 